\title{
Review of: "Microglia do not restrict SARS-CoV-2 replication following infection of the central nervous system of K18-hACE2 transgenic mice"
}

\author{
Vijay Kumar \\ 1 The University of Tennessee Health Science Center
}

Potential competing interests: The author(s) declared that no potential competing interests exist.

I found this article interesting as authors have tried to show that microglia are not required for exerting protective antiviral immune response in the brain of K18-hACE2 transgenic mice. Although, these microglia were secreting potential pro-inflammatory cytokines and chemokines during SARS-CoV-2 infection by day 7 post infection as the microglia depletion decreased their levels. These authors observed highest levels of CCL9 and CXCL10 (T cell chemoattractants), CCL2 and CCL5 (monocyte and macrophage chemoattractants), and TNF-alpha along with CCL19 (B cell chemoattractant), IFN-lambda type 3 and IL-10. This indicates that the microglia activation in patients with SARS-CoV-2 infection or COVID-19 may increase the pro-inflammatory damage to neurons through secreting different pro-inflammatory molecules (cytokines and chemokines) that attract pro-inflammatory T cells, B cells, and monocytes/macrophages. These authors have also showed that SARS-CoV-2 infected human neuron do not show syncytia formation that shows that lack of virus spread to the adjacent neurons through cell fusion. The neurons infected with SARS-CoV-2 showed the over activation of elF2 signaling pathway along with oxidative phospshorylation (OXPHOS), elF4 and mTOR signaling (preventing autophagy but increasing exaggerated protein synthesis) that may induce cell death. The intranasal infection with the SARS-CoV-2 showed the neuroinvasion along with inducing the pneumonia as observed in human patients of COVID-19. Hence, this study has shown the non-protective action of microglia during SARS-CoV-2 infection that exerts anitviral action in other viral infection. Further studies will help to explore the exact mechanism associated through genetic tools-based targeting of microglia. 